\title{
Thoracic Trauma
}

\author{
Loren Ketai and Steven L. Primack
}

\section{Learning Objectives}

- Understand how the CT spectrum of ATAI influences treatment decisions.

- Know the imaging appearance of the most common survivable cardiac injuries.

- Describe the differences in imaging findings between blunt and penetrating diaphragm injuries.

- Know the coexisting injury patterns associated with fractures and dislocations involving the sternum and shoulder girdle.

Both blunt and penetrating traumas commonly affect the thorax, blunt trauma occurring predominantly as the result of motor vehicle collisions. The thorax is the fourth most injured area in unrestrained motor vehicle passengers but is the most commonly injured area in individuals who are restrained by a seat belt [1]. Other common causes of blunt thoracic trauma include falls from heights of greater than 10 feet and motor vehicle collisions involving pedestrians/ bicyclists.

While not as common as blunt thoracic injury, penetrating thoracic trauma is not rare. Records from the Centers for Disease control (CDC) show that in 2016, the deaths from gunshot wounds in the United States were comparable to the number of deaths from motor vehicle accidents (MVAs). Tissue damage from these injuries may be complex and is dependent not only on the trajectory of the projectile but

\section{Ketai}

Department of Radiology, University of New Mexico HSC, Albuquerque, NM, USA

e-mail: 1ketai@unm.edu

\section{S. L. Primack $(\bowtie)$}

Department of Diagnostic Radiology, Oregon Health and Science University, Portland, OR, USA

e-mail: primacks@ohsu.edu upon the pressure gradient that radiates laterally from the tract causing temporary cavitation within adjacent tissue [2]. In both blunt and penetrating trauma, radiologists' familiarity with typical injury patterns assists with rapid diagnosis and treatment.

While the initial screening test in thoracic trauma is the frontal chest radiograph obtained in the trauma bay, in many emergency rooms, this is accompanied by E-FAST (extended focused assessment with sonography in trauma) performed by non-radiologists. In addition to detecting intraabdominal trauma, this study can detect important thoracic injuries, specifically pneumothoraces, hemothoraces, and hemopericardium. Patients with large hemothoraces or hemopericardium can be directed to emergent surgery, while the remaining patients with significant trauma mechanism should usually be further evaluated by CT [3]. Among patients with penetrating trauma, CT with multiplanar reconstructions is particularly useful to determine the trajectory of a bullet through the chest and identify injured organs. Used in hemodynamically stable patients, CT can accurately identify those patients that can be managed nonoperatively, reported negative predictive values as high as $99 \%$ [4].

\subsection{Acute Traumatic Aortic Injury}

Acute traumatic aortic injury (ATAI) is a common cause of prehospital mortality in MVAs, accounting for approximately $15-20 \%$ of deaths in high-speed collisions. Of individuals with ATAI, 70-90\% die at the trauma scene [5]. Most of the surviving 10-20\% individuals with ATAI from blunt trauma undergo CT imaging. Patients with penetrating aortic trauma who reach the hospital usually proceed to surgery without intervening imaging.

Decision rules using clinical parameters for predicting ATAI have had very limited success. An initial case control study restricted true positive cases to those confirmed by surgery or conventional angiography. In that population, the 
likelihood of ATAI was approximately 5\% if three clinical predictors were present and $30 \%$ if four or more predictors were present. A more recent case control validation study performed by the same group in the era of CT angiography identified only four composite clinical criteria that were predictive of ATAI (abdominopelvic injury, thoracic injury, hypotension, and history of MVA while unrestrained). The likelihood of ATAI in patients with three or four of these criteria was only $2 \%$, much lower than the derivation study, and is probably more relevant to current clinical practice [6].

Among patients surviving to reach the hospital, $90 \%$ of blunt aortic injuries are seen at the level of the aortic isthmus, which extends from the origin of the subclavian artery approximately $2 \mathrm{~cm}$ to the insertion of the ligamentum arteriosum. The propensity for injury to occur at this location is incompletely understood, and multiple mechanisms have been proposed. The ascending aorta is injured in less than $5 \%$ of blunt ATAI victims who survive transport, but injuries at that site are more common at autopsy series [5]. Ascending aorta injuries are frequently $(80 \%)$ associated with significant cardiac damage, including damage to the aortic valve or pericardial tamponade. Traumatic aortic injuries located at the diaphragmatic hiatus account for less than $2 \%$ of cases.

The continuous motion of the heart during cross-sectional scanning leads to motion artifacts of the ascending aorta that can have the appearance of aortic dissection or pseudoaneurysm in non-cardiac-gated acquisitions, even with current rapid acquisition multidetector CT scanners. These artifacts can often be differentiated from intimal flaps, appearing less distinct and sometimes extending outside the vessel wall.

Because less than $5 \%$ of surviving patients will have an ascending aortic injury, the benefit of gated cardiac CT at initial presentation is limited. Most patients with ATAI are too unstable to undergo prolonged imaging, and thus evaluation of the aorta with standard, nongated contrast-enhanced CT is generally preferred. In patients whose initial CT contains artifacts closely mimicking aortic root injury, cardiacgated CT can be performed.

Transesophageal echocardiography is a potential alternative to CTA for exclusion of ATAI if there are no contraindications, such as vertebral body fracture or severe maxillofacial injury. The disadvantages of transesophageal echocardiography include the logistical time required to perform the study and its user dependence as well as inability to evaluate the remainder of the thorax.

ATAI ranges from intimal hemorrhage to complete transection. The adventitia may be injured in up to $40 \%$ of cases and is almost universally fatal because of rapid exsanguination, although in rare cases, temporary tamponade may be achieved by surrounding mediastinal soft tissues. Historically, of those patients who survive to the hospital after aortic rupture, $22 \%$ die during initial resuscitation, $28 \%$ die during or shortly after surgical repair, and 14\% develop subsequent paraplegia [7]. Shift of current practice to endovascular stenting has been associated with post-procedure mortality rates $<10 \%$ and a decrease in the incidence of paraplegia to $<2 \%[8]$.

\subsubsection{Radiography}

Although the principal purpose of the trauma bay chest radiograph is the rapid detection of pneumothorax and hemothorax, it can also be assessed for evidence of a mediastinal bleeding. This bleeding can be seen secondary to ATAI, sternal/thoracic spine fracture, or small artery or vein damage within the mediastinum. The resulting mediastinal blood can be free or partially free flowing (associated with small mediastinal vessel rupture) or form a contained hematoma which is more concerning for an ATAI.

The negative predictive value of a properly obtained chest radiograph may be as high as $93-98 \%$, but consideration of mechanism severity is important before ATAI can be excluded. The positive predictive value of an abnormal radiograph remains low, only $15 \%$. Specificity of mediastinal widening is degraded by mediastinal fat, vascular congestion, anomalous vessels, and rotation. When the right paratracheal region is equal to or more opaque than the aortic arch or a left apical cap is present, mediastinal widening is more likely to represent a free-flowing mediastinal blood [9].

Radiographic findings more suggestive of a contained aortic rupture include rightward deviation of an enteric tube, rightward deviation of the trachea, downward displacement of the left main bronchus, loss of aortic arch definition, increased aortic arch opacity, and increased width and opacity of the descending aorta [9].

\subsubsection{Computed Tomography}

Multidetector helical CT is a very sensitive diagnostic tool for exclusion of ATAI, often quoted as the gold standard for ATAI detection [10]. A morphologically normal aorta with no mediastinal hematoma has a $100 \%$ negative predictive value for exclusion of ATAI. The positive predictive value of an equivocal study (i.e., para-aortic hematoma only) is uncertain; these studies require additional imaging or clinical follow-up. Mediastinal hematomas not in contact with the aorta, but located in the anterior or posterior mediastinum, are more likely to be related to chest wall or spinal fractures [11]. If the patient is a victim of penetrating trauma with a wound trajectory passing near the aorta, a repeat CTA should be obtained in 24-48 h.

The direct signs of ATAI on CT include aortic wall or contour abnormality (pseudoaneurysm) contrast extravasation (Fig. 12.1), polypoid intraluminal clot or low-density filling 

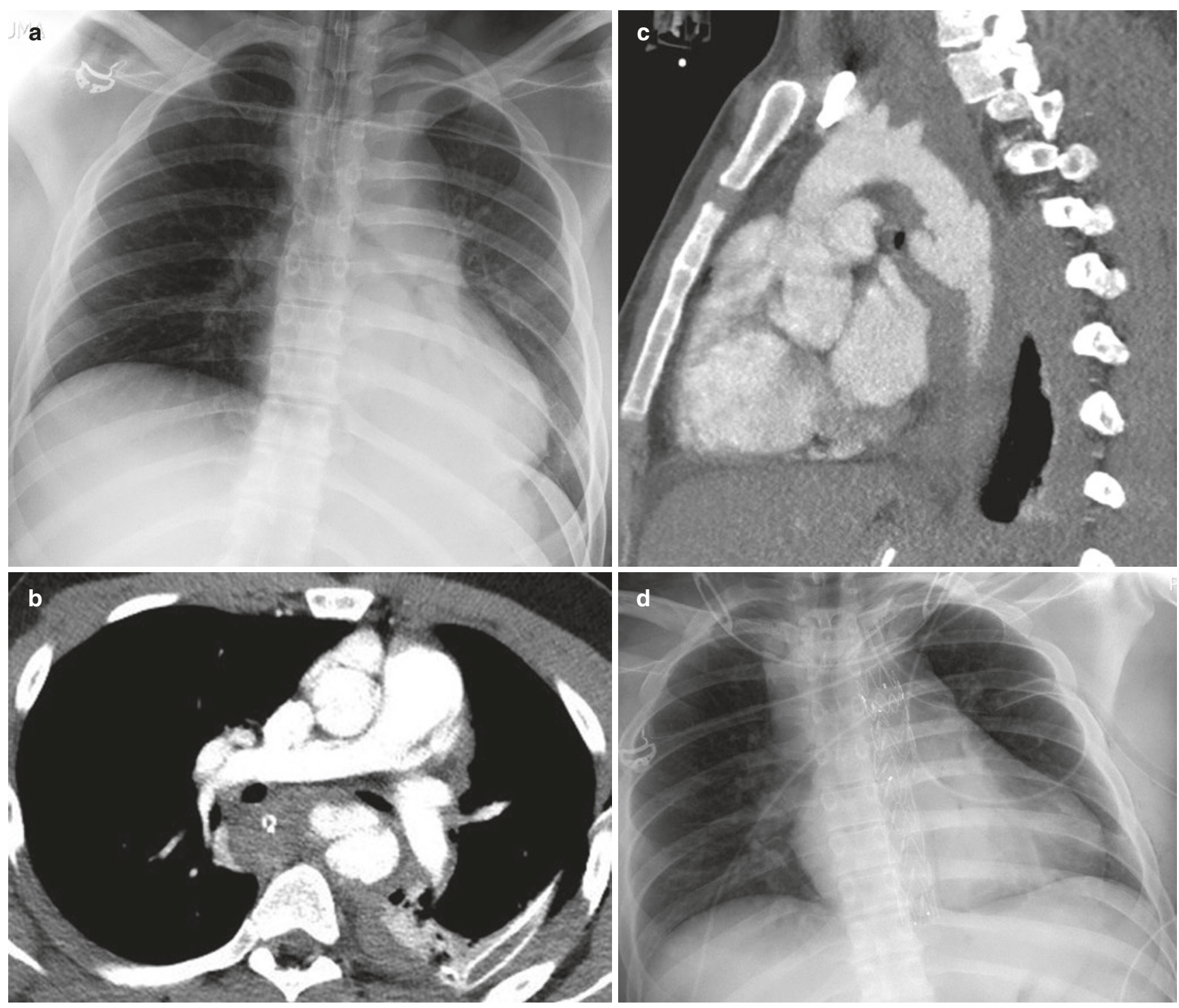

Fig. 12.1 Traumatic aortic injury. (a) AP chest radiograph shows widened mediastinum and indistinctness of aortic arch contour. (b, c) Axial (b) and sagittal (c) contrast-enhanced CT demonstrates disruption of

defect, change in caliber of the aorta (i.e., pseudocoarctation), and dissection [10]. The least severe injury, intimal tear, appears on contrast-enhanced CT as either a rounded or triangular intraluminal filling defect attached to the wall of the aorta.

Recently the surgical treatment of ATAI has become more nuanced, and reporting of ATAI should include details useful for surgical decision-making. Initially nonoperative therapy was focused on "minimal" aortic injuries that were sometimes described as small $(<1 \mathrm{~cm})$ intimal flaps (Fig. 12.2). More recently initial nonoperative therapy has been extended to intimal injuries extending over more than $1 \mathrm{~cm}$ and to intramural hematomas [12]. These intramural hematomas may involve the entire circumference of the aortic wall or occur within a portion of the aortic wall, such that the high- the proximal descending thoracic aortic wall contour with contrast extravasation indicating aortic rupture. (d) AP chest radiograph following intervention with aortic stent

attenuation thrombus appears crescentic in shape. Neither intimal injuries nor intramural hematomas, classified as grade I and II in the surgical literature, are accompanied by an abnormal outer contour of the aorta. If initial management is nonoperative, follow-up CT imaging is necessary. Exact timing of follow-up is not well established, but a reasonable approach would be a repeat CT in 36-72 $\mathrm{h}$ and then again in several weeks if stable [13]. A minority of these injuries demonstrate progression on follow-up CT imaging, at which time endovascular stenting can be performed if necessary.

Unlike lower-grade injuries described above, pseudoaneurysm do cause an abnormal outer contour abnormality. Small pseudoaneurysms (less than $1 \mathrm{~cm}$ in length and less than $50 \%$ of the aortic diameter) may also be initially treated nonoperatively in hemodynamically stable patients 

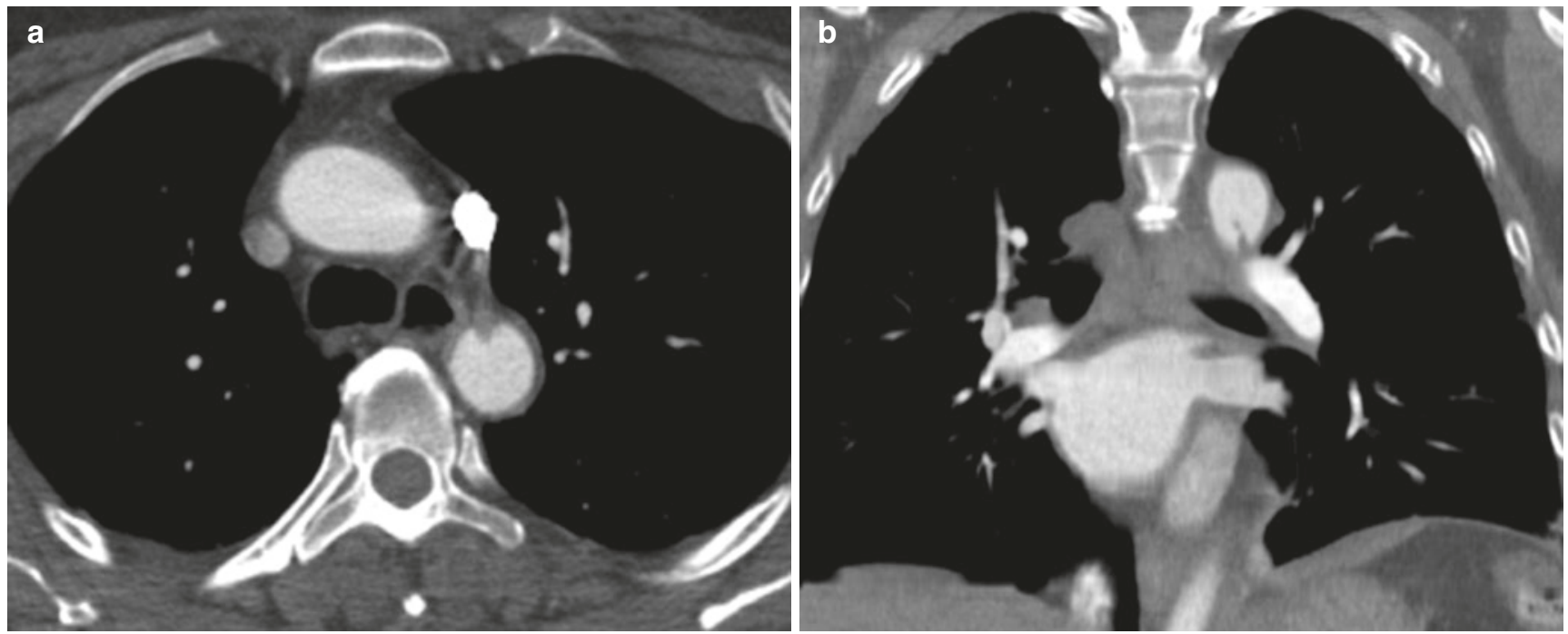

Fig. 12.2 Minimal traumatic aortic injury. (a, b) Axial (a) and coronal (b) contrast-enhanced CT demonstrates an intimal flap involving the proximal descending thoracic aorta

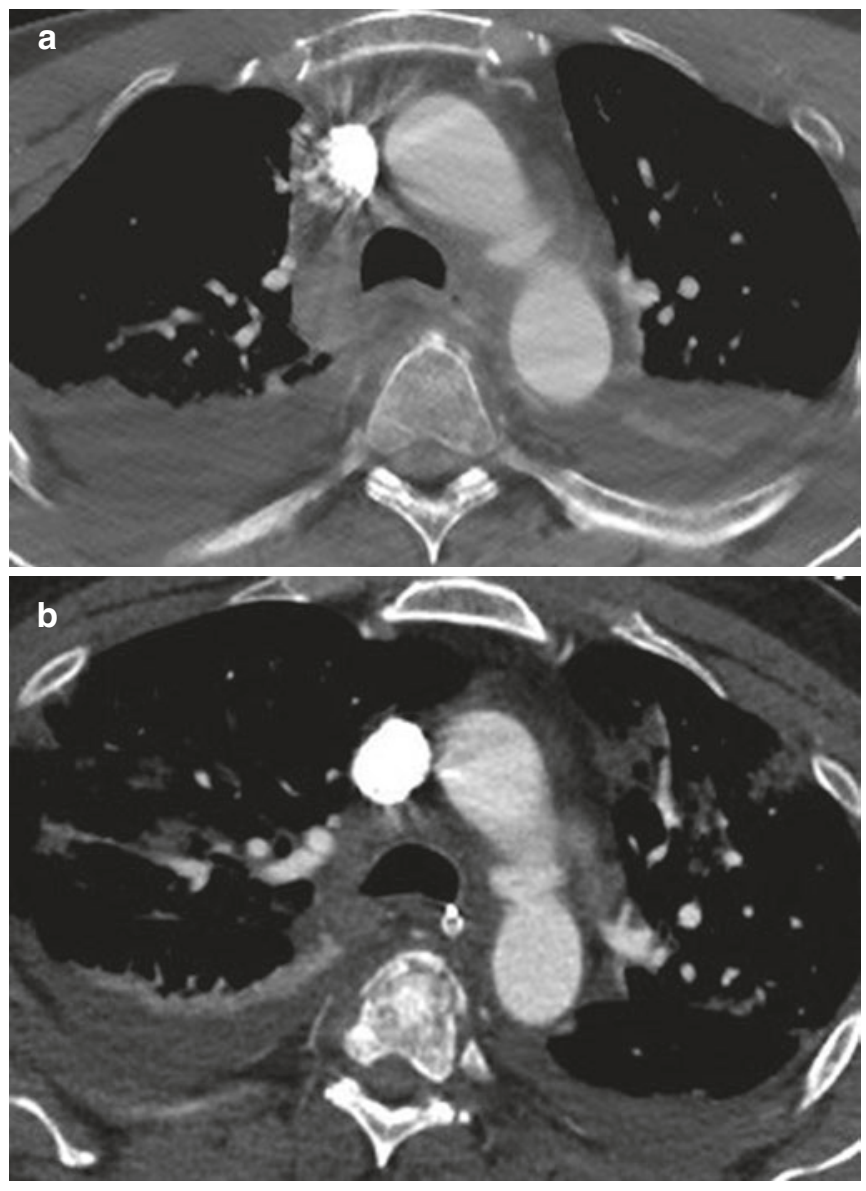

Fig. 12.3 Minimal traumatic aortic injury. (a) Axial contrast-enhanced CT demonstrates a small pseudoaneurysm arising from the undersurface of the aortic arch. (b, c) 2 weeks following presentation, axial (b)

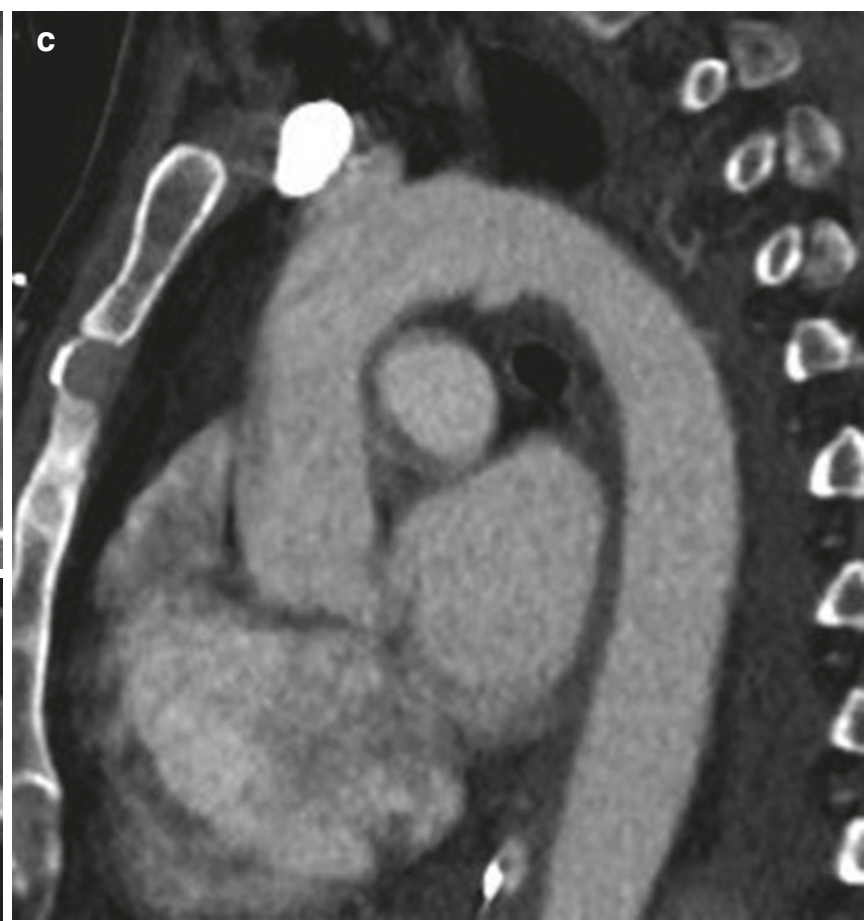

and sagittal (c) contrast-enhanced CT show no change in small pseudoaneurysm 
(Fig. 12.3). Those pseudoaneurysms that involve greater than $50 \%$ of the aortic circumference or are accompanied by an adjacent mediastinal hematoma $>15 \mathrm{~mm}$ thick are at higher risk for progression and may be difficult to differentiate from aortic ruptures. These injuries warrant more urgent intervention. True thoracic aortic rupture/transection remains a surgical emergency.

\section{Key Points}

Report Elements to Include on CTAs Positive for TAI

- Presence of intimal flap and its length

- Presence of endoluminal thrombus

- Presence of outer contour abnormality

- Extent of involvement of the aortic circumference (e.g., <50\%)

- Length of abnormality along the axis of the aorta (e.g., $<1 \mathrm{~cm})$

- Distance of proximal extent of injury from the subclavian artery (relevant for TEVAR)

\subsection{Great Vessel and Supra-aortic Branch Vessel Injury}

Injuries to the superior and inferior vena cava, the pulmonary artery, and the pulmonary veins are commonly associated with other severe, non-survivable injuries and therefore are rarely imaged. When encountered, SVC ruptures are associated with right atrial rupture and azygous vein ruptures and are accompanied by mediastinal hematoma [14]. Blunt trauma injuries to the supra-aortic branch vessels, the brachiocephalic artery (right subclavian/right common carotid), left common carotid artery, and the left subclavian artery are also uncommon. In one retrospective series of blunt trauma, ATAI made up $84 \%$ of the vascular injuries, isolated branch vessel injury $14 \%$, and combined injuries only $4 \%$ [15].

Penetrating trauma with potential injury to the great vessels and supra-aortic vessels often involves a transmediastinal trajectory of the projectile. These are frequently severe injuries, and hemodynamically unstable patients usually proceed to surgery with minimal imaging. Patients who are hemodynamically stable may undergo echocardiography or E-Fast exam followed by CTA. Since transmediastinal penetrating trauma can involve the esophagus and tracheobronchial tree, exams optimally include upper alimentary tract contrast (see below).

\subsubsection{Radiography}

The screening trauma radiograph is unlikely to aid in the diagnosis of great vessel and supra-aortic branch vessel injury, other than to identify ballistic trajectory in the setting of penetrating trauma. As in ATAI, a mediastinal hematoma is usually present.

\subsubsection{Computed Tomography}

Similar to the setting of ATAI, CT may demonstrate intimal flaps and pseudoaneurysms. Alternatively, subtle findings, such as asymmetric vascular caliber or decreased contrast enhancement, may provide evidence of vascular injury. Although multidetector CT scans are likely to detect a traumatic injury to the supra-aortic branch vessel, catheter angiography maintains a role in confirming injury [15].

CT findings caused by penetrating vascular trauma include those signs of injury described above, as well formation of arteriovenous fistula. Additional indirect findings include proximity to ballistic fragments and end organ infarcts due to peripheral embolization of intraluminal thrombus. As for blunt injuries, many vessel abnormalities seen on CTA warrant catheter-directed angiography for confirmation and possible treatment.

\subsection{Cardiac Injury}

Significant cardiac injuries are uncommon in blunt trauma victims surviving to reach the emergency department. Autopsy data indicate that numerous prehospital deaths result from traumatic cardiac or valvular rupture. Penetrating injury to the heart is traditionally suspected when wounds penetrate the "cardiac box," an area on the anterior thorax bounded by the clavicles, the midclavicular lines, and the costal margin where intersected by the midclavicular lines. Recent literature has defined the cardiac box in three dimensions, extending anterior-posterior, particularly in the left hemithorax.

Blunt cardiac injury can cause cardiac contusion and more critical injuries specifically pericardial, myocardial, or valvular rupture. Myocardial injuries most commonly involve the right ventricular free wall, while valvular injuries more commonly occur in the left-sided chambers, likely related to higher transvalvular pressures. The most innocuous and most common of these injuries, cardiac contusion, is usually diagnosed based on troponin levels and ECG findings. Although CT can reveal multiple ancillary signs of cardiac contusion, myocardial enhancement is an insensitive indicator and should not be used to rule out cardiac injury [16]. Echocardiogram and occasionally MRI may aid diagnosis of cardiac contusion by evaluating for wall motion abnormalities.

Hemopericardium may be the result of cardiac chamber rupture (usually fatal prior to arrival in the hospital), myocar- 
dial contusion, aortic root injury, coronary lacerations, or damage to the small surrounding pericardial vessels such as those damaged from displaced rib fractures. Pericardial rupture is a rare injury. It commonly occurs on the left side, often along the course of the phrenic nerve, and can be accompanied by tears of the adjacent pleura or diaphragm. Such injuries become hemodynamically significant when the heart herniates through the tear, impeding cardiac filling and potentially causing coronary vessel occlusion. The proposed mechanism of injury for pericardial rupture likens the injury to a pendulum since the inferior portion of the heart is free floating without significant attachments. In the setting of a lateral deceleration injury, the heart presumably collides with the adjacent pericardium, creating the rupture [17]. Cardiac herniation through a pericardial defect may not be present initially and may occur after the administration or discontinuation of positive-pressure ventilation.

The most commonly ruptured cardiac chamber in the autopsy of blunt trauma victims is the right ventricle, while the atria are the most commonly ruptured chambers in individuals who survive to hospitalization. Penetrating trauma most commonly affects the right ventricle, followed in frequency by the left ventricle. Right ventricle and right atrial wounds are more likely to be survivable than left-sided chambers. Unfortunately, the increased relative incidence of gunshot wounds compared to stabbing injuries over the last two decades has been associated with multichamber cardiac injuries, which have a much lower survival rate.

\subsubsection{Radiography}

Hemopericardium may or may not be evident on chest radiograph at presentation. Chest radiography may show an unusual position or configuration of the heart if cardiac herniation is present. As noted above, in the setting of penetrating trauma, chest radiographs can yield important clues to the projectile's trajectory.

\subsubsection{Computed Tomography}

Most patients who have a mechanism of injury sufficient to produce hemopericardium are imaged with $\mathrm{CT}$ if stable. Simple fluid should demonstrate attenuation values of $0-10$ $\mathrm{HU}$, whereas hemopericardium will have attenuation values in the $35 \mathrm{HU}$ range. The rapid accumulation of hemopericardium may result in cardiac tamponade, the diagnosis suggested when CT demonstrates a triad of high-attenuation pericardial effusion, distention of the inferior vena cava and renal veins, and periportal low-attenuation fluid. Hemopericardium is visible on nearly all CT scans in cases of pericardial rupture. Not all pericardial tears are associated with cardiac herniation; the chest radiograph and CT scan may be unrevealing. Pericardial rupture is diagnosed on CT by the presence of a focal pericardial defect with a collar sign of herniated cardiac tissue. The presence of adjacent pneumothorax, pneumomediastinum, or both may complicate the diagnosis if the pericardium is mistaken for the pleura.

CT does not play a diagnostic role in patients with penetrating injury that are either hemodynamically unstable or demonstrate hemopericardium on ultrasound. As with transmediastinal penetrating injuries, CT may be useful in establishing bullet trajectory in hemodynamically stable patients and suggest occult cardiac injury based on the projectile track or the presence of ballistic fragments. CT may demonstrate occult cardiac trauma in 3-4\% of hemodynamically stable patients with penetrating thoracic trauma, usually by demonstrating hemopericardium or pneumopericardium [18].

\subsection{Pneumomediastinum}

Pneumomediastinum is present in $10 \%$ of patients with blunt thoracic injury and is most commonly secondary to the Macklin effect [19]. This is the process by which air dissects along bronchovascular bundles after alveolar rupture and spreads to the mediastinum. Mediastinal air may also occur from the extension of subcutaneous or deep cervical emphysema. Direct extension of retroperitoneal air to the mediastinum occurs via periesophageal and periaortic fascial planes and at the level of the sternocostal attachment of the diaphragm [9].

\subsubsection{Radiography}

Pneumomediastinum can be identified on the chest radiograph by visualization of lucency along the pericardium and lateral mediastinum outlined laterally by the pleura [9]. Pneumomediastinum may be difficult to differentiate from medial pneumothorax; this can best be done by searching for other manifestations of the two entities. Air within the mediastinum characteristically outlines other structures so that they are more conspicuous than usual. These include the "ring around the artery sign" (air surrounds right pulmonary artery), "tubular artery sign" (air outlines supra-aortic vessels), and "double bronchial wall sign" (air outlines the outer contour of a major bronchus) [20]. Air posterior to the pericardium yields the continuous diaphragm.

Differentiation of pneumomediastinum from pneumopericardium on radiography relies on recognition that air is not confined to the limits of the pericardium, an assessment most 


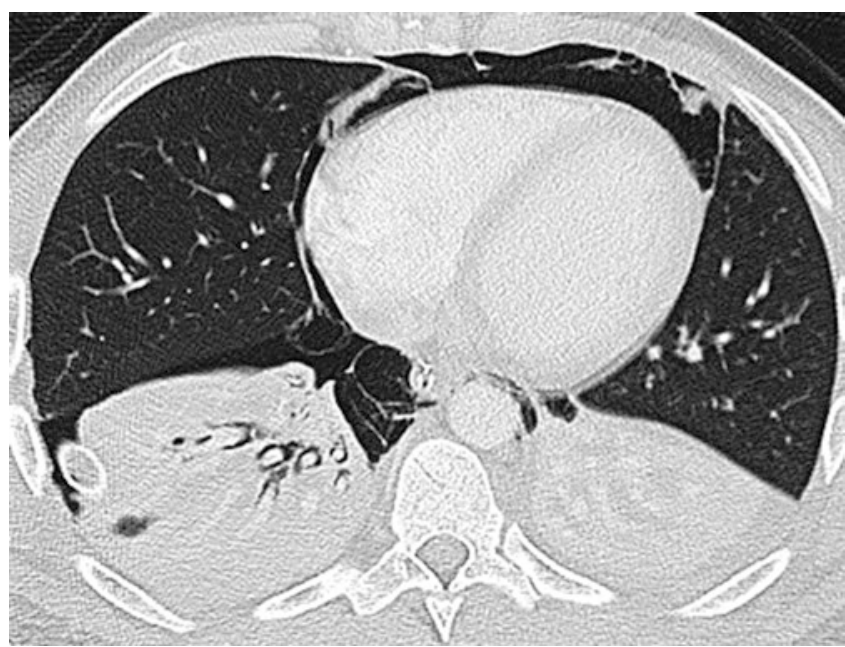

Fig. 12.4 Pneumomediastinum due to Macklin effect following gunshot wound to the right lung. Axial CT demonstrates extensive pneumomediastinum secondary to alveolar rupture. Note peribronchovascular interstitial air in the right lower lobe. Macklin effect is most commonly related to blunt trauma or barotrauma

commonly confirmed by identifying subcutaneous emphysema in the neck. An additional pitfall is the error in perception caused by Mach effect, which causes an apparent lucency surrounding the convexity of the heart border that mimics pneumomediastinum. The absence of an adjacent opaque pleural line usually differentiates this from true pneumomediastinum.

Pneumomediastinum typically resolves with time. Serious complications, such as tension pneumomediastinum, are rare. Failure of resolution or lack of improvement with time raises the suspicion of tracheobronchial or penetrating esophageal injury. Esophageal rupture from blunt trauma is rare.

\subsubsection{Computed Tomography}

CT can prove the presence of Macklin effect by demonstrating the triad of a bronchus, a pulmonary vessel, and an additional adjacent air collection (Fig. 12.4). The presence of Macklin effect does not exclude the possibility of a tracheobronchial or esophageal injury, though both are unlikely if the resulting pneumomediastinum is confined to the anterior compartment. The absence of the Macklin effect calls for increased scrutiny of the CT images to identify signs of esophageal or airway injury (see below).

\subsection{Esophageal Injury}

Esophageal injuries caused by non-iatrogenic trauma are rare but potentially catastrophic. Mortality for undiagnosed rupture after $24 \mathrm{~h}$ is $10-40 \%$. Chest radiograph findings include pneumomediastinum and pleura effusions, left sided if the lower third of the esophagus is injured, right side if upper two thirds is injured. Fluoroscopic esophagography is moderately sensitive, e.g., $75 \%$, and very specific but can be technically difficult to perform in critically ill patients. CT esophagography (CTE) performed after ingestion or instillation of contrast and effervescent granules was shown to be $100 \%$ sensitive in detecting injury in a small group of patients. A more recent study following a similar protocol with the exception of effervescent granules has reported a sensitivity near $80 \%$. In the setting of penetrating trauma, concomitant performance of CTA and CTE may diminish specificity [21].

\subsection{Airway Injury}

Tracheobronchial injuries include mucosal tears and complete transection of the airway. These are uncommon injuries, occurring in $1-3 \%$ of patients with blunt thoracic trauma.

\subsubsection{Radiography}

The radiographic manifestations of tracheobronchial injury are usually nonspecific and include pneumomediastinum, pneumothorax, deep cervical emphysema, and subcutaneous emphysema. Injuries to the trachea or proximal left main bronchus typically result in pneumomediastinum but not pneumothorax. The fallen lung sign is specific to bronchial fracture. This sign describes the lung "falling" dependently, rather than collapsing centrally toward the hilum in the presence of a pneumothorax.

Tracheal or bronchial tear should be suspected with persistent or increasing soft tissue emphysema, or if pneumothorax persists despite continuous chest tube drainage. Extraluminal position of the endotracheal tube and cuff overinflation also indicate tracheal injury [22].

\subsubsection{Computed Tomography}

Approximately $75 \%$ of tracheobronchial injuries occur within $2 \mathrm{~cm}$ of the carina. Right main bronchus injuries generally occur closer to the carina (usually within $2.5 \mathrm{~cm}$ ) than injuries to the left main bronchus, and the right main bronchus is injured more frequently than either the trachea or the left main bronchus. While CT can readily demonstrate secondary signs of trachea bronchial injury (pneumomediastinum and pneumothorax), older literature demonstrated relatively low sensitivity in detection of direct evidence of 

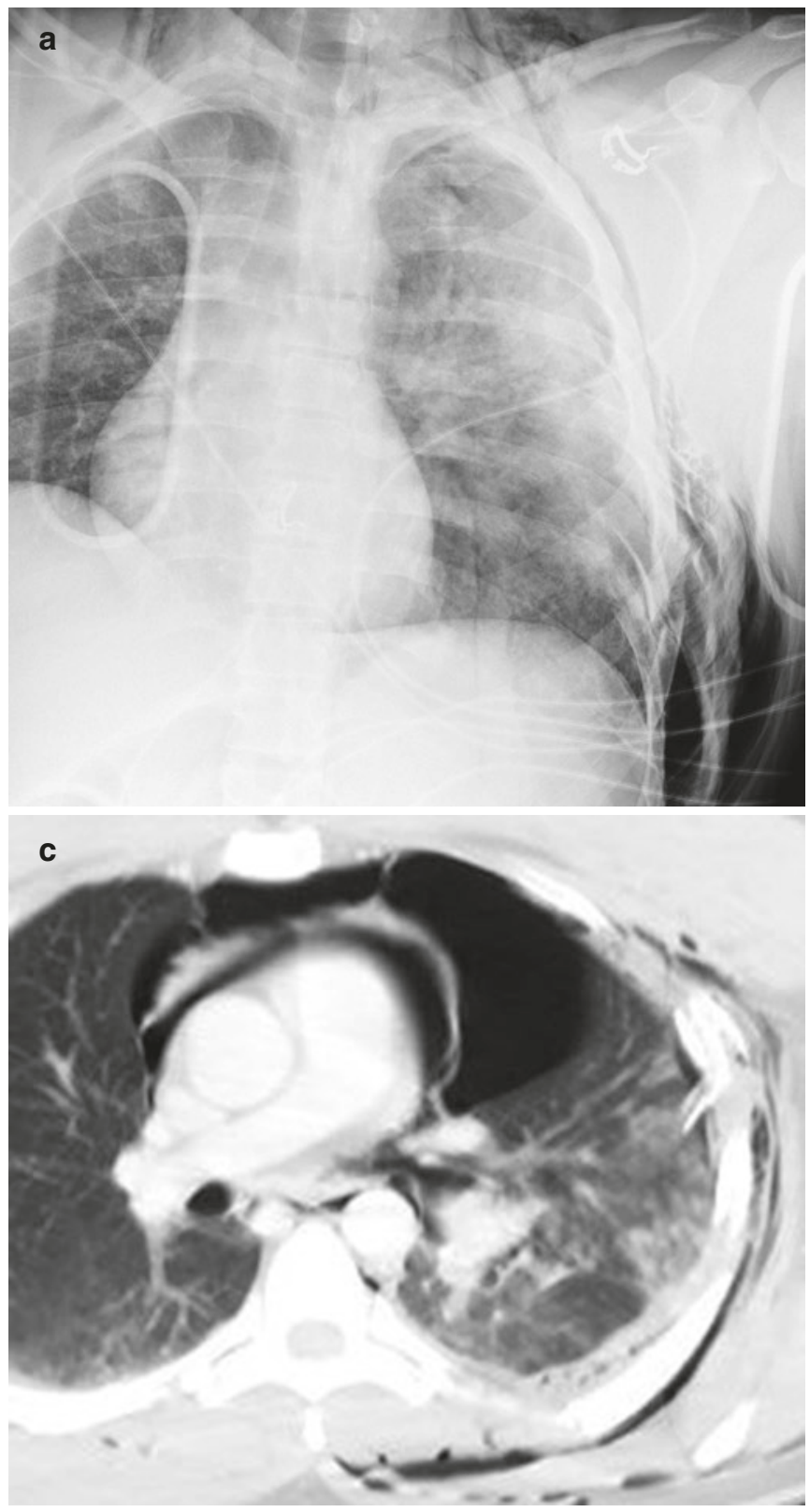

Fig. 12.5 Left bronchial tear. (a) AP chest radiograph shows extensive predominantly left-sided subcutaneous air, pneumomediastinum and left rib fractures. (b) CT at the level of the main bronchi shows extensive pneumomediastinum, left pneumothorax and subcutaneous air. (c)

tracheobronchial injuries [23]. Direct visualization of a tear on CT can be difficult and best accomplished using thin $(<3$ $\mathrm{mm}$ ) collimation (Fig. 12.5). More recent small series using thinner collimation have reported sensitivities $>90 \%$ [24]. Nevertheless complications of tracheobronchial injury can be severe, including bronchopleural and tracheoesophageal fistulas, and chronic tracheal or bronchial stenosis leading to postobstructive pneumonia and bronchiectasis. Accordingly, a high index of suspicion and liberal use of bronchoscopy are important for the prompt diagnosis of tracheobronchial injury.

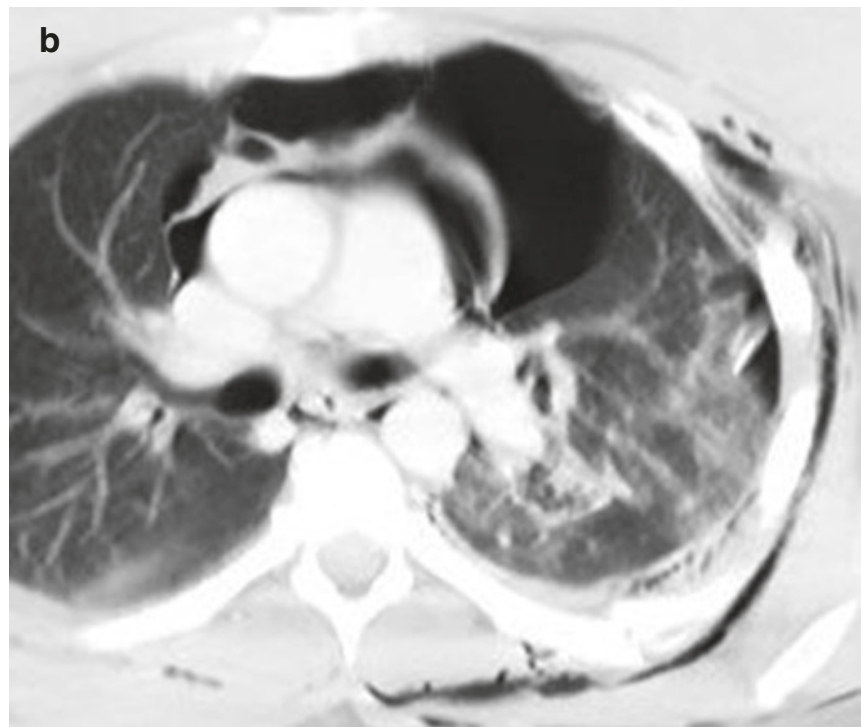

CT inferior to the level of (b) demonstrates complete disruption of leftsided airways. At surgery, there was near complete disruption of the distal left main bronchus with tear extending into the left upper and lobe bronchi

\subsection{Pulmonary Contusion}

Pulmonary contusion is seen in $30-70 \%$ of patients with blunt thoracic injury $[1,25]$. It is the most common cause of pulmonary parenchymal opacification on chest radiography in blunt thoracic trauma. Short-term morbidity is substantial, and clinical signs and symptoms include hypoxia, mild fever, hemoptysis, and dyspnea. Acute respiratory failure may develop, requiring intubation and mechanical ventilation. Contusion may also be associated with long-term respiratory dysfunction. 
Pulmonary contusion represents pulmonary hemorrhage and edema from disruption of the alveolar capillary membrane [9]. It is often caused by a direct blow to the chest wall with injury of the adjacent lung and may be accompanied by contrecoup injuries. Contusion is frequently seen where the lungs have been compressed against the denser heart, liver, chest wall, and spine. In deceleration injuries, such as motor vehicle accidents, the alveolar capillary membrane is disrupted as the lower-density alveoli shear from the higherdensity bronchovascular bundles $[9,26]$.

\subsubsection{Radiography}

Pulmonary contusions can manifest as focal, patchy, or diffuse ground-glass opacities or parenchymal consolidation. These opacities cross fissural boundaries and are often present at the site of impaction and adjacent to rib fractures. The lung bases are most frequently affected secondary to increased basilar mobility [9]. Air bronchograms are usually absent owing to blood filling the airways.

Pulmonary parenchymal contusion follows a predictable temporal pattern on imaging. Contused lung may not be apparent on initial chest radiography but is usually present within $6 \mathrm{~h}$ after the inciting injury. Conspicuity of pulmonary opacities peaks at 24-72 $\mathrm{h}$ and gradually diminishes over 1 week although severe contusions may be radiographically evident for 2 weeks. Persistence beyond this duration should prompt consideration of other pulmonary diseases, such as pneumonia, atelectasis, aspiration pneumonitis, and hydrostatic or noncardiogenic pulmonary edema.

\subsubsection{Computed Tomography}

$\mathrm{CT}$ is superior to chest radiography in the detection of pulmonary contusion, but the clinical utility of this higher sensitivity is likely low. Contusions visible on CT but not on chest radiograph are associated with low mortality and low likelihood of requiring mechanical ventilation, both $<5 \%$. CT-based quantification of more extensive contusions may be helpful in predicting respiratory complications [27]. For example, the likelihood of development of ARDS is much higher in those patients with contusion involving $>20 \%$ of the lung. CT may also be helpful in distinguishing acute aspiration from contusion, tree in bud opacities being present in the former and absent in pulmonary contusions. CT demonstration of subpleural sparing by parenchymal opacities (Fig. 12.6) has been shown to accurately differentiate pulmonary contusions from pneumonia and atelectasis in children.

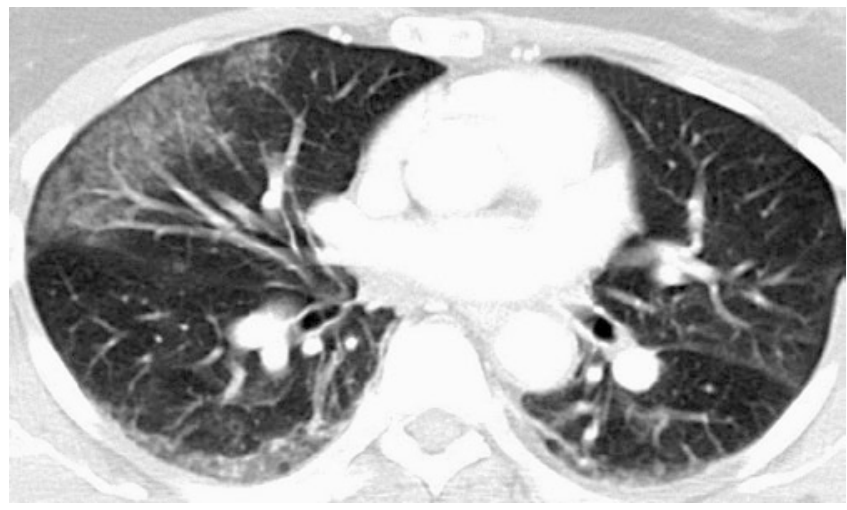

Fig. 12.6 Pulmonary contusion. Axial CT demonstrates peripheral homogeneous ground-glass opacity anteriorly in the right middle lobe. Note the thin region of subpleural sparing

\subsection{Pulmonary Laceration and Traumatic Lung Cyst}

Lacerations of the lung parenchyma cause traumatic lung cysts that may be filled with air (pneumatocele), blood (hematoma), or both. Several mechanisms of injury in blunt trauma have been proposed based on CT and surgical findings. Type 1 , the most common type, occurs when a sudden compressive force causes alveolar rupture. Type 2 results from shear injury of the lower lung near the spine as a compressive force causes the lung to shift across the vertebral column. Type 3 manifests as lung cysts near the chest wall adjacent to a rib fracture fragment that has directly punctured the pulmonary parenchyma. Type 4 occurs when previously formed pleuropulmonary adhesions tear the lung after sudden movement or fracture of the attached chest wall.

\subsubsection{Radiography}

In approximately half of the cases, pulmonary lacerations are obscured by the consolidation related to pulmonary contusion on the initial chest radiograph. As the lung contusion resolves, pulmonary lacerations become visible as thinwalled cystic spaces or as soft tissue nodules (pulmonary hematomas). Radiographic resolution of traumatic lung cysts occurs over weeks to months. Uncommonly, the cysts persist for years. If clot remains in the cavity, these cysts can be mistaken for pulmonary nodules, and air-filled cavities may mimic other forms of cystic lung disease. Knowledge of previous trauma is important in correctly differentiating between these entities. 


\subsubsection{Computed Tomography}

On CT imaging, traumatic lung cysts manifest as ovoid structures secondary to the elastic recoil of the lung [9]. Blood may completely fill the cavity causing an ovoid pulmonary hematoma or can partially fill the cavity, causing an air-fluid level is present. Large cystic spaces may be present, or blunt trauma can create multiple, small, air-filled lung cysts sometimes referred as "pulverized" or "Swiss cheese" appearance of the lungs [26]. The most common complication associated with a pulmonary laceration from blunt is a pneumothorax, and, while rare, other complications of traumatic lung cysts include infection with formation of a pulmonary abscess and the development of a bronchopleural fistula.

Lung lacerations commonly occur secondary to penetrating trauma and can manifest as linear lung defects along the projectile track or as round or oval air cysts. Like blunt trauma, penetrating trauma to the lungs usually can be managed with chest tube placement. Occasionally treatment of bronchopleural fistula or hemorrhage requires surgery, most commonly pneumonorraphy, tractotomy, or wedge resection, rarely lobectomy or pneumonectomy [28].

Acute Lung Parenchymal Trauma

- Pulmonary contusion

- Peak extent in 24-72 h

- Peripheral, nonanatomic opacities, sometimes with immediate subpleural sparing

- Lung lacerations

- Pneumatoceles appear as thin-walled cystic

Unilocular or multilocular

With or without air-fluid levels

- Hematomas manifest as soft tissue masses that may persist for months.

- Acute aspiration

- Tree in bud opacities, dependent lung or diffuse

- May progress to ARDS

\section{Key Points} spaces.

rib fractures and/or flail chest. It presents as new onset sharp pleuritic chest pain and dyspnea. Occasionally, intraperitoneal blood migrates to the pleural space via diaphragmatic defects or rupture of the diaphragm.

\subsubsection{Radiography}

Small hemothoraces may be missed easily on chest radiography. On the supine radiograph, the apex is the most dependent portion of the pleural space, and fluid should be suspected if an apical cap is seen. Larger amounts of fluid will extend inferiorly along the lateral chest wall. On supine radiographs fluid also may manifest as a hazy opacity of the lower or entire hemithorax.

\subsubsection{Computed Tomography}

Hemorrhage within the pleural space typically can be differentiated from simple fluid or chyle by its relatively high, and often heterogeneous, attenuation, usually 35-70 HU. On contrast-enhanced CT, fluid with attenuation within 10-15 HU of vascular structures suggests active extravasation. Occasional findings include fluid-hematocrit levels and coalescence of blood clots that may mimic pleural-based tumors. CT can also be useful in quantifying retained hemothoraces, which are associated with high rates of pneumonia and empyema. Volume of residual hemothorax can be estimated by the following formula: volume $=$ maximal length $\times$ (maximal distance from lung to pleural surface) [2]. Residual hemothorax $>300$ cc usually requires video-assisted thoracoscopy (VATS) or thoracotomy [29].

\subsection{Pneumothorax}

Pneumothorax is a common sequela of blunt chest trauma. Mechanisms include rupture of the alveoli and dissection through the interstitium to the pleural space, lung puncture from rib fracture fragments, tracheobronchial injury, and extension of pneumomediastinum through a tear in the parietal pleura [9, 25]. Small pneumothoraces can expand rapidly in patients receiving positive-pressure ventilation.

\subsubsection{Radiography}

Hemothorax occurs in approximately $50 \%$ of blunt thoracic trauma. Intrathoracic blood may arise from any surrounding structures, including the lung, mediastinum, thoracic wall, and diaphragm [26]. Hemothoraces may be large and increase rapidly if the bleeding source is arterial. Delayed hemothorax occurs rarely but is more common in patients with multiple
The definitive radiographic finding of pneumothorax, a thin pleural line seen when air is present on either side of the visceral pleura, is often absent on supine radiographs. On supine radiographs air often accumulates in the anteromedial recess, the least dependent aspect of the pleural space 
and in the subpulmonic recess. Signs of anteromedial pneumothorax include sharp delineation of the mediastinal structures, outlining of the medial diaphragm beneath the heart, and a deep anterior cardiophrenic sulcus. A subpulmonic pneumothorax manifests as a hyperlucent upper abdominal quadrant, deep lateral costophrenic sulcus, and visualization of the anterior costophrenic sulcus and inferior surface of the lung. Subcutaneous air coupled with rib fractures indicates the presence of pneumothorax even if pleural air is radiographically occult.

Tension pneumothorax is a life-threatening condition that occurs when intrathoracic pressure exceeds atmospheric pressure, causing hemodynamic compromise. Mild mediastinal shift is commonly present in the absence of tension, since the negative pleural pressure in the unaffected hemithorax will always be lower than in the pleural space affected by the pneumothorax. The most specific radiographic finding of tension pneumothorax is depression or inversion of the diaphragm, which indicates that pleural pressure has exceeded subdiaphragmatic pressure [9].

\subsubsection{Computed Tomography}

CT is much more sensitive than radiography in the detection of pneumothoraces, $30 \%$ of which are missed on supine and semirecumbent chest radiographs [9]. Prospective study has suggested that many pneumothoraces seen on CT only may be managed without tube thoracostomy, even if on positive pressure ventilation [30]. Risk of tension pneumothorax has diminished with the current use of lower tidal volumes for mechanical ventilation, but this complication can still occur.

\subsection{Traumatic Diaphragmatic Injury}

Traumatic diaphragmatic tears can result from penetrating or blunt injury. Penetrating diaphragmatic injury is twice as common as blunt injury. Diaphragmatic rupture occurs in $0.8-8 \%$ of all patients with major blunt trauma [9]. Detection of diaphragmatic injury requires a high index of suspicion since masking of the injury by pleural fluid or pulmonary contusion may delay diagnosis. In addition, diaphragmatic injury is associated with other major traumatic injuries, including pneumothorax, hepatic and splenic lacerations, and pelvic fractures, which may distract from identification of diaphragmatic injury [31].

\subsection{Mechanism of Injury}

Blunt frontal impact can cause sufficient increase in intraabdominal pressure to rupture the diaphragm. Shearing injury may occur when a lateral blow distorts the chest wall [31].
Diaphragmatic tears associated with blunt trauma are usually $10 \mathrm{~cm}$ or greater. Injury is most frequently left-sided $(75 \%)$ owing to the protective cushioning effect of the liver. Relative difficulty in detection of right-sided injury may contribute to its underdiagnosis and underrepresentation in the literature.

Penetrating injury results from shootings, stabbings, and, less commonly, iatrogenic causes such as a malpositioned chest tube. Stabbings and shootings usually result in leftsided diaphragmatic damage, presumably because most assailants are right-handed and facing their victims. Entrance wound occurring anywhere between the level of the nipples and inferior costal margin have the potential to cross the diaphragm. The resulting focal diaphragmatic injury often produces a small tear in the diaphragm (usually $<1 \mathrm{~cm}$ in length). CT detection of penetrating diaphragm injury has increased in importance as more patients with penetrating trauma (most commonly stabbing victims) undergo nonoperative management, such that laparotomy cannot be relied upon to discover small diaphragm defects. Occult diaphragm injury has been found in up to $20 \%$ of patients with penetrating trauma who would otherwise be managed nonoperatively [32].

\subsubsection{Radiography}

The chest radiograph, although a useful screening tool, is relatively insensitive in the detection of diaphragmatic rupture. Overall sensitivity ranges from $17 \%$ to $64 \%$, although serial radiographs can increase sensitivity by $12 \%$ [10]. Radiographic signs of diaphragmatic injury include obscuration of the hemidiaphragm, elevation of the hemidiaphragm, and contralateral shift of the mediastinum [31]. The most specific radiographic finding of diaphragmatic injury is the presence of gas-containing stomach or bowel within the thorax. Termination of a nasogastric tube within the left hemithorax is another specific finding.

\subsubsection{Computed Tomography}

CT using thin collimation and thin reconstruction images (1to 2-mm-thick slices) is the imaging modality of choice in the evaluation of suspected diaphragmatic injury in patients with blunt or penetrating trauma. Sagittal and coronal reformations have been shown to increase sensitivity and confidence of interpretation. Sensitivities of 70-90\% are reported.

A constellation of CT findings has been evaluated for the detection of diaphragmatic rupture. The most frequently cited are direct discontinuity or focal defect of the diaphragm, intrathoracic herniation of abdominal viscera, the collar sign, and the dependent viscera sign (Fig. 12.7). Visualization of a focal discontinuity of the diaphragm is $73-82 \%$ sensitive and $90 \%$ specific for diaphragmatic injury. Bochdalek hernias, focal defects in the posterolateral dia- 

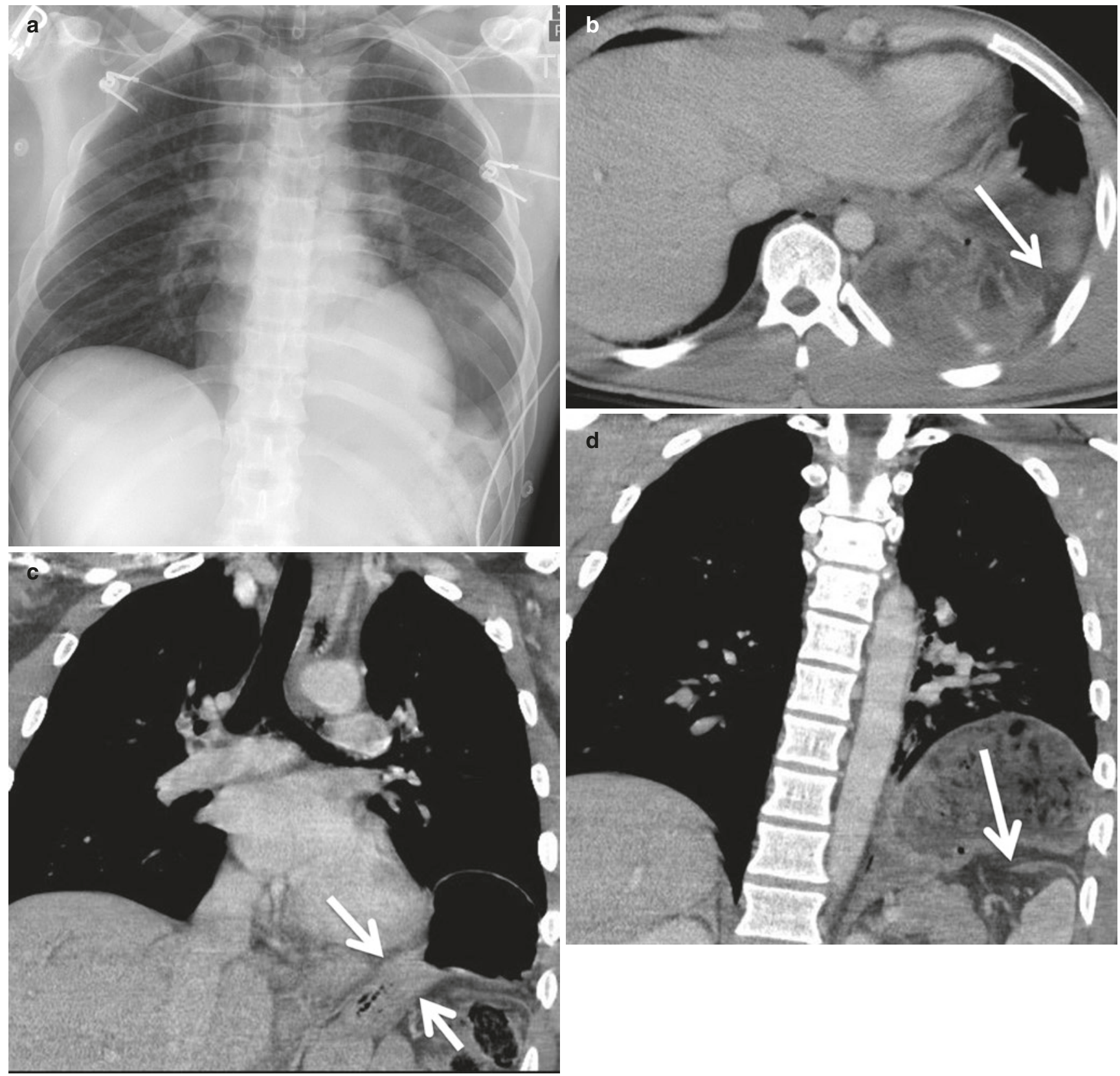

Fig. 12.7 Traumatic diaphragmatic injury with herniation. (a) AP chest radiograph shows an elevated left hemidiaphragm with stomach projecting in the lower left hemithorax. (b) Axial contrast-enhanced CT demonstrates stomach displaced posteriorly (dependent viscera sign)

phragm, may mimic traumatic rupture and account for falsepositive findings. Intrathoracic herniation has a sensitivity of $55-75 \%$ and a specificity of $100 \%$ [33]. Waist-like constriction of herniated abdominal contents at the level of diaphragmatic rupture creates the collar sign, best depicted on coronal and sagittal reformations. With thin section helical CT, the collar sign has a sensitivity of $63 \%$ and a specificity of $100 \%$. The dependent viscera sign describes the position of the stomach, spleen, or bowel that has herniated into the thorax

and suggestion of a waist-like constriction (arrow). (c, d) Coronal CT images demonstrate herniation of a portion of the stomach into the thorax and depict the collar sign (arrows in (c)) and abrupt discontinuity of left hemidiaphragm (arrow in (d))

and lies adjacent the dependent aspect of the chest wall. This sign yields sensitivity of 55-90\% for blunt trauma diaphragmatic injuries. Other signs suggestive of diaphragmatic injury include thickening and segmental non-visualization of the diaphragm [33].

Penetrating diaphragm injuries tend to be considerably smaller than blunt diaphragmatic injuries, and accordingly radiologic signs based on the herniation of abdominal viscera are only occasionally helpful. Diagnosis depends on 
demonstration of a simple breach of the diaphragm. Direct visualization of the diaphragm defect on $\mathrm{CT}$ is specific but uncommonly seen. CT demonstration of contiguous injuries on both signs of the diaphragm is the most sensitive CT finding and in many cases and its absence can be used to exclude injury. Unfortunately, its specificity can be diminished if multiple wounds are present. Construction of double oblique CT images along the trajectory of the knife or bullet (trajectography) can improve specificity of diagnosis. Demonstration of a tract extending on both sides of the diaphragm is specific for penetrating diaphragm injury and in many cases is adequate impetus for operative exploration [32].

\section{Key Points}

CT Findings in Diaphragmatic Tear

- Blunt

Injury Findings (Sensitivity of CT - 70-90\%)

- Sharp discontinuity of hemidiaphragm

- Herniation of omental fat or abdominal viscera

- Waist-like narrowing of herniated viscera (collar sign)

- Lack of visualization of hemidiaphragm

- Dependent viscera sign

- Penetrating

Injury Findings (Sensitivity of CT - 80-100\%)

- Contiguous injury - single wound

- Contiguous injury - multiple wounds (decreased specificity)

- Transdiaphragmatic trajectory

\subsection{Thoracic Skeletal or Chest Wall Trauma}

Rib fractures have prognostic significance and can by themselves cause physiologic impairment. In general rib fractures are more common in elderly individuals, due to lesser elasticity of the skeleton. In children, on the other hand, rib fractures indicate a significant mechanism of injury and are associated with a higher mortality rate [1].Rib fractures are uncommon in restrained passengers in collisions at speeds of less than 30 miles $(<50 \mathrm{~km})$ per hour [1] but overall occur in $60 \%$ of patients with blunt thoracic trauma. The most commonly fractured ribs are the fourth through the ninth ribs. Mortality increases slightly in patients with two or more rib fractures visible on CT but increases more sharply when nine or more are visible.

Several patterns of rib fractures are associated with specific injuries. Fractures of the 10th, 11th, and 12th ribs are associated with renal, splenic, and hepatic injuries. First, second, and third ribs are associated with a significant mech- anism of injury and are accompanied by aorta or great vessel injury in $14 \%$ and tracheobronchial injuries in $2 \%$ of cases [34]. Rib fractures accompanied by disruption of chest wall soft tissues can result in lung herniation. In the setting or blunt trauma, this occurs most commonly anteriorly or posteriorly which layers of intercostal muscles are fewest. Patients with greater than four continuous ribs that are fractured in two placed (segmental fractures) are at increased risk for development of a flail chest.... This is a clinical diagnosis in which an unstable portion of the chest wall moves paradoxically during respiration, impairing ventilation.

Sternal fractures are seen predominately in restrained passengers and occur in $8 \%$ of individuals with a significant mechanism of injury. The presence of sternal fractures is associated with severity of chest injury, the degree of displacement being the major determinant. Less than $10 \%$ of patient with minimally displaced sternal fractures have spine fractures or traumatic pericardial effusions. Patients with completely displaced sternal fractures, however, have $>40 \%$ chance of having a traumatic pericardial effusion and $>30 \%$ chance of having a spine fracture. Although associated with cardiac injury, sternal fractures are not associated with ATAI.

Location of fracture within the sternum is also significant, with fractures involving the manubrium having the highest association with spine fractures, usually involving the upper thoracic spine (T5-T6) and the thoracolumbar junction (T12L1) [35]. Sternomanubrial dislocations share this association with thoracic spine trauma, specifically type 2 dislocations in which the manubrium is displaced posteriorly with respect to the sternal body. This type of dislocation is caused by hyperflexion, the same mechanism often fracturing the thoracic spine. Type-one sternomanubrial dislocations in which the sternal body is displaced posteriorly with respect to the manubrium are usually the result of a direct blow, rather than hyperflexion. Internal mammary arteries can be directly damaged by this posterior displacement of the sternal body.

The sternoclavicular joints and sternum (see above) are important sites to evaluate for fracture or dislocation because injury in these locations can cause adjacent mediastinal hematoma that may raise concern for ATAI if the associated fracture is not identified. Sternoclavicular dislocations are also clinically relevant due to potential direct damage to adjacent structures. These injuries are rare due to extensive ligamentous support, such that forces transmitted along the clavicle are far more likely to fracture the clavicle than dislocate the joint. Anterior sternoclavicular dislocation is the most common type of dislocation, while posterior dislocation is associated with more severe sequelae, such as tracheal, esophageal, and great vessel impingement or injury.

Scapular fractures indicate a significant mechanism of injury and can be seen on $57 \%$ of initial chest radiographs [34]. Approximately 50\% of patients with a scapular fracture have a pneumothorax. Ipsilateral subclavian, axillary, or brachial artery injury may be present in $11 \%$ of patients with 
scapular fracture [1]. Vascular and neurologic injury are also associated with scapulothoracic disassociations, a more severe injury that separates the scapula and muscular attachments of upper extremity from the thorax without disrupting the overlying skin.

Thoracic spine fractures occur in 3\% of blunt trauma patients and are most common in the thoracolumbar region [34]. Wedge compression and burst fractures secondary to hyperflexion and axial load mechanisms are the predominant fracture types [36] and frequently result in significant neurologic impairment. Neurologic impairment is common because the spinal cord occupies a larger percentage of the available space in the canal than in the more spacious cervical and lumbar spine regions. Additionally, thoracic cord blood supply has little collateral reserve, predisposing to ischemic insult.

\subsubsection{Radiography and Computed Tomography}

CT is more sensitive than chest radiography in the diagnosis of rib and may be used to three-dimensional reformatted images to better visualizing rib trauma. In the setting of a flail chest, this may aid the surgeon in surgical planning. Fixation of rib fractures in the setting of flail segment may decrease the patient duration of mechanical ventilation, but the evidence is not strong, and operative fixation is not commonly performed [37]. Peripheral opacities that parallel the chest wall represent extrapleural hematomas adjacent to rib fractures. Extrapleural hematoma forms superficial to the parietal pleura, arising from bleeding from damaged intercostal vessels, and muscles. These extrapleural hematomas can be identified on CT by the presence of inward displacement of extrapleural fat and are not treated by tube thoracostomy. Collections that are biconvex may represent an arterial bleeding source and are more likely to require surgical intervention [38]. The possibility of a flail chest should also be considered whenever a large extrapleural hematoma is seen.

Sternal fractures are rarely seen on frontal radiographs but, similar to rib fractures, are readily seen on CT images, particularly sagittal reconstructions. Diagnosis of sternoclavicular dislocation without $\mathrm{CT}$ is also very difficult, even with special radiographic views (e.g., serendipity view).

Radiographic diagnosis of scapulothoracic disassociation relies on detecting scapular displacement via the scapula index, a measurement easily distorted by difference in arm positioning and by rotation. In suspected cases CTA is essential to diagnose occlusion of subclavian and axillary vessels from thrombosis or external compression. MRI is best suited for evaluating associated brachial plexus injuries; the presence of $>3$ pseudomeningoceles suggests irreversible nerve root damage.

\section{Key Points}

- Sternal fracture.

- Completely displaced-traumatic pericardial effusion (40\%) and spine fracture $(30 \%)$

- Minimally displaced-traumatic pericardial effusion/spine fracture $(10 \%)$

- Manubrium fracture

- Thoracic spine (T5-T6) and the thoracolumbar junction (T12-L1)

- Sternomanubrial dislocation

- Type 2-hyperflexion spinal injury

- Type 1-internal mammary artery injury

- Posterior sternoclavicular joint dislocation

- Brachiocephalic vein and aortic arch vessels

- Scapulothoracic disassociation

- Subclavian/axillary artery, brachial plexus, and pseudomeningoceles

\subsection{Concluding Remarks}

Over the last two decades, cross-sectional imaging of both blunt and penetrating thoracic traumas has helped clinicians select from among widened options for injury management. Advances in CT imaging and evolution of treatment for ATAI now allow for nonoperative treatment of some injuries and delayed endovascular stent placement for others. Conversely, the presence of signs of penetrating diaphragmatic injury may prompt laparotomy in patients initially triaged to nonoperative management. Despite these improvements in CT imaging, diagnostic uncertainty may still prompt invasive procedures for some injuries (e.g., catheter angiography for supra-aortic vessel injury, bronchoscopy for suspected large airway rupture).

\section{Take-Home Messages}

- Patients with minimal ATAI are managed nonoperatively. In the remaining ATAI victims, accurate description of pseudoaneurysm extent may facilitate management with either imaging follow-up or non-emergent endovascular stenting rather than emergent intervention.

- CT demonstration of hemopericardium is a nonspecific finding that can be due to pericardial injury, cardiac contusion, and rarely rupture of right-sided cardiac chambers. Focal constriction of cardiac chambers suggests underlying pericardial rupture, while accompanying IVC distention and periportal edema are indicators of associated tamponade. 
- Manubrial fracture and posterior dislocation of the manubrium are strongly associated with spine fractures, while posterior sternal body dislocation, posterior sternoclavicular dislocations, and scapulothoracic disassociation prompt careful evaluation for vascular injuries.

- Blunt diaphragmatic injuries cause large defects that are detectable by direct visualization, displacement of viscera, or deformation of herniated contents (collar sign). Detection of the smaller defects caused by penetrating trauma depends on determining the trajectory of the penetrating object.

\section{References}

1. Mayberry J. Imaging in thoracic trauma: the trauma surgeon's perspective. J Thorac Imaging. 2000;15:76-86.

2. Durso A, Caban K, Munera F. Penetrating thoracic injury. Radiol Clin N Am. 2015;53:675-93.

3. Schellenberg M, Inaba K, Bardes JM, Orozco N, Chen J, Park C, et al. The combined utility of extended focused assessment with sonography for trauma and chest x-ray in blunt thoracic trauma. J Trauma Acute Care Surg. 2018;85(1):113-7.

4. Strumwasser A, Chong V, Chu E, Victorino GP. Thoracic computed tomography is an effective screening modality in patients with penetrating injuries to the chest. Inj Int $\mathrm{J}$ Care Injured. 2016;47:2000-5.

5. Steenburg SD, Ravenel JG, Ikonomidis JS, Schönholz C, Reeves S. Acute traumatic aortic injury: imaging evaluation and management. Radiology. 2008;248(3):748-62.

6. Kirkham JR, Blackmore CC. Screening for aortic injury with chest radiography clinical factors. Emerg Radiol. 2007;14:211-7.

7. Cowley RA, Turney SZ, Hankins JR, et al. Rupture of thoracic aorta caused by blunt trauma. A fifteen-year experience. J Thorac Cardiovasc Surg. 1990;100:652.

8. Morgan TA, Steenburg SD, Siegel EL, Mirvis SE. Acute traumatic aortic injuries: posttherapy multidetector CT findings. Radiographics. 2010;30:851-67.

9. Costantino M, Gosselin MV, Primack SL. The ABC's of thoracic trauma imaging. Semin Roentgenol. 2006;41:209-25.

10. Raptis C, Hammer M, Raman K, et al. Acute traumatic aortic injury: practical considerations for the diagnostic radiologist. J Thorac Imaging. 2015;30:202-13.

11. Rojas CA, Restrepo CS. Mediastinal hematomas: aortic injury and beyond. J Comput Assist Tomogr. 2009;33:218-24.

12. Gunn ML, Lehnert BE, Lungren RS, et al. Minimal aortic injury of the thoracic aorta: imaging appearances and outcome. Emerg Radiol. 2014;21:227-33.

13. Harris DG, Rabin J, Starnes BW, Khoynezhad A, Conway G, et al. Evolution of lesion-specific management of blunt thoracic aortic injury. J Vasc Surg. 2016;64:500-5.

14. Holly BP, Steenburg SD. Multidetector CT of blunt traumatic venous injuries in the chest, abdomen, and pelvis. Radiographics. 2011;31(5):1415-2.
15. Chen MY, Miller PR, McLaughlin CA, Kortesis BG, Kavanagh PV, Dyer RB. The trend of using computed tomography in the detection of acute thoracic aortic and branch vessel injury after blunt thoracic trauma: single-center experience over 13 years. J Trauma. 2004 Apr;56(4):783-5.

16. Hammer MM, Raptis DA, Cummings KW, et al. Imaging in blunt cardiac injury: computed tomographic findings in cardiac contusion and associated injuries. Injury. 2016;47(5):1025-30.

17. Restrepo CS, Gutierrez FR, Marmol-Velez JA, Ocazionez D, Martinez-Jimenez S. Imaging patients with cardiac trauma. Radiographics. 2012;32:633-49.

18. Plurad DS, Bricker S, Van Natta TL, Neville A, Kim D, et al. Penetrating cardiac injury and the significance of chest computed tomography findings. Emerg Radiol. 2013;20:279-84.

19. Wintermark M, Schnyder P. The Macklin effect: a frequent etiology for pneumomediastinum in severe blunt chest trauma. Chest. 2001;120:543-7.

20. Zylak CM, Standen JR, Barnes GR, Zylak CJ. Pneumomediastinum revisited. Radiographics. 2000;20:1043-57.

21. Conradie JW, Gebremariam FA. Can computed tomography esophagography reliably diagnose traumatic penetrating upper digestive tract injuries? Clin Imaging. 2015;39(6):1039-45.

22. Cassada DC, Munyikwa MP, Monitz MP, et al. Acute injuries of the trachea and major bronchi: importance of early diagnosis. Ann Thorac Surg. 2000;69:1563-7.

23. Kunisch-Hoppe M, Hoppe M, Rauber K, Popella C, Rau WS. Tracheal rupture caused by blunt chest trauma: radiological and clinical features. Eur Radiol. 2000;10:480-3.

24. Scaglione M, Romanoa S, Pinto A, Sparano A, Scialpi MB, Rotondo A. Acute tracheobronchial injuries: impact of imaging on diagnosis and management implications. Eur J Radiol. 2006;59:336-43.

25. Rivas LA, Fishman JE, Múnera F, Bajayo DE. Multislice CT in thoracic trauma. Radiol Clin N Am. 2003;41:599-616.

26. Mille r LA. Chest wall, lung, and pleural space trauma. Radiol Clin N Am. 2006;44:213-24.

27. Rodriguez RM, Friedman B, Langdorf MI, Baumann BM, Nishijima DK, Hendey GW, Medak AJ, Raja AS, Mower WR. Pulmonary contusion in the pan-scan era. Injury. 2016;47(5):1031-4.

28. Huh J, Wall MJ Jr, Estrera AL, Soltero ER, Mattox KL. Surgical management of traumatic pulmonary injury. Am J Surg. 2003;186:620-4.

29. DuBose J, Inaba K, Demetriades D, Scalea TM, O'Connor J, Menaker J, et al. Management of post-traumatic retained hemothorax: a prospective, observational, multicenter AAST study. J Trauma Acute Care Surg. 2012 Jan;72(1):11-22.

30. Moore FO, Goslar PW, Coimbra R, Velmahos G, Brown CV, Coopwood TB Jr, et al. Blunt traumatic occult pneumothorax: is observation safe?-results of a prospective, AAST multicenter study. J Trauma. 2011;70(5):1019-23.

31. Iochum S, Ludig T, Walter F, et al. Imaging of diaphragmatic injury: a diagnostic challenge? Radiographics. 2002;22:S103-18.

32. Dreizin D, Bergquist PJ, Taner AT, Bodanapally UK, Tirada N, Munera F. Evolving concepts in MDCT diagnosis of penetrating diaphragmatic injury. Emerg Radiol. 2015;22:149-56.

33. Nchimi A, Szapiro D, Ghaye B, et al. Helical CT of blunt diaphragmatic rupture. Am J Roentgenol. 2005;184:24-30.

34. Collins J. Chest wall trauma. J Thorac Imaging. 2000;15:112-9.

35. von Garrel T, Ince A, Junge A, Schnabel M, Bahrs C. The sternal fracture: radiographic analysis of 200 fractures with special reference to concomitant injuries. J Trauma. 2004;57:837-44. 
36. Kuhlman JE, Pozniak MA, Collins J, Knisely BL. Radiographic and CT findings of blunt chest trauma: aortic injuries and looking beyond them. Radiographics. 1998;18:1085-106.

37. Kasotakis G, Hasenboehler EA, Streib EW, Patel N, Patel MB, Alarcon L, Bosarge PL, Love J, Haut ER, Como JJ. Operative fixation of rib fractures after blunt trauma: a practice management guideline from the Eastern Association for the Surgery of Trauma. J Trauma Acute Care Surg. 2017;82(3):618

38. Chung JH, Carr RB, Stern EJ. Extrapleural hematomas: imaging appearance, classification, and clinical significance. J Thorac Imaging. 2011;26:218-23.

Open Access This chapter is licensed under the terms of the Creative Commons Attribution 4.0 International License (http://creativecommons. org/licenses/by/4.0/), which permits use, sharing, adaptation, distribution and reproduction in any medium or format, as long as you give appropriate credit to the original author(s) and the source, provide a link to the Creative Commons license and indicate if changes were made.

The images or other third party material in this chapter are included in the chapter's Creative Commons license, unless indicated otherwise in a credit line to the material. If material is not included in the chapter's Creative Commons license and your intended use is not permitted by statutory regulation or exceeds the permitted use, you will need to obtain permission directly from the copyright holder. 